\title{
BMJ Global Health Inequities in the incidence and safety of abortion in Nigeria
}

\author{
Suzanne O Bell, ${ }^{1}$ Elizabeth Omoluabi, ${ }^{2}$ Funmilola OlaOlorun, ${ }^{3}$ Mridula Shankar, ${ }^{1}$ \\ Caroline Moreau (iD ${ }^{1}$
}

To cite: Bell SO, Omoluabi E, OlaOlorun $\mathrm{F}$, et al. Inequities in the incidence and safety of abortion in Nigeria. BMJ Global Health 2020;5:e001814. doi:10.1136/ bmjgh-2019-001814

\section{Handling editor Sanni Yaya}

Received 2 July 2019 Revised 14 October 2019 Accepted 10 November 2019

Check for updates

(c) Author(s) (or their employer(s)) 2020. Re-use permitted under CC BY-NC. No commercial re-use. See rights and permissions. Published by BMJ.

${ }^{1}$ Population Family and Reproductive Health, Johns Hopkins University Bloomberg School of Public Health, Baltimore, Maryland, USA ${ }^{2}$ Centre for Research, Evaluation Resources and Development, Ile-Ife, Nigeria

${ }^{3}$ Department of Community Medicine, University of Ibadan, Ibadan, Oyo, Nigeria

Correspondence to Professor Suzanne 0 Bell; suzanneobell@gmail.com

\section{ABSTRACT}

Background We know little about the frequency, correlates and conditions under which women induce abortions in Nigeria. This study seeks to estimate the 1-year induced abortion incidence and proportion of abortions that are unsafe overall and by women's background characteristics using direct and indirect methodologies.

Methods Data for this study come from a populationbased, nationally representative survey of reproductive age women (15-49) in Nigeria. Interviewers asked women to report on the abortion experiences of their closest female confidante and themselves. We adjusted for potential biases in the confidante data. Analyses include estimation of 1-year induced abortion incidence and unsafe abortion, as well as bivariate and multivariate assessment of their correlates.

Results A total of 11106 women of reproductive age completed the female survey; they reported on 5772 confidantes. The 1-year abortion incidence for respondents was 29.0 (95\% Cl 23.3 to 34.8) per 1000 women aged $15-49$ while the confidante incidence was 45.8 (95\% $\mathrm{Cl}$ 41.0-50.6). The respondent and confidante abortion incidences revealed similar correlates, with women in their 20s, women with secondary or higher education and women in urban areas being the most likely to have had an abortion in the prior year. The majority of respondent and confidante abortions were the most unsafe $(63.4 \%$ and $68.6 \%$, respectively). Women aged 15-19, women who had never attended school and the poorest women were significantly more likely to have had the most unsafe abortions.

Conclusion Results indicate that abortion in Nigeria is a public health concern and an issue of social inequity. Efforts to expand the legal conditions for abortion in Nigeria are critical. Simultaneously, efforts to increase awareness of the availability of medication abortion drugs to more safely selfinduce can help mitigate the toll of unsafe abortion-related morbidity and mortality.

\section{BACKGROUND}

In Nigeria, abortion is only legal to save a woman's life. Recent estimates extrapolated from facility-based abortion complications indicate there were approximately 33 abortions per 1000 women aged $15-49$ in $2012{ }^{1}$ This is equivalent to 1.25 million abortions annually, representing more than half $(56 \%)$ of all unintended pregnancies to Nigerian

\section{Key questions}

What is already known?

- The most recent research on induced abortion in Nigeria suggested there were 33 abortions per 1000 women aged 15-49 in 2012; however, these data lack information regarding the social determinants of abortion or unsafe abortion as the estimates rely largely on a facility-level measure of complications and do not include women's characteristics.

What are the new findings?

- Results indicate there were 45.8 abortions per 1000 women of reproductive age in 2018, approximately two-thirds of which were unsafe.

- Young, educated and urban women were more likely to have had a recent abortion while young, uneducated, rural and poor women were more likely to have had an unsafe abortion.

\section{What do the new findings imply?}

- These findings illustrate that abortion is very common in Nigeria and that unsafe abortion is an issue of health inequity, with the most disadvantaged women most likely to experience an unsafe abortion.

women. These predominantly unsafe abortions resulted in nearly 500000 women experiencing serious health consequences, less than half (212 000) of whom received treatment for these complications. ${ }^{1}$ The only previous national study of abortion incidence in Nigeria estimated a rate of 25 abortions per 1000 women aged 15-44 in 1996, suggesting that women's use of abortion as a means of fertility control has increased in intervening years. $^{2}$

Recent regional estimates of abortion safety indicate that nearly $85 \%$ of abortions in West Africa are considered unsafe. ${ }^{3}$ These unsafe abortions are a result of women seeking clandestine procedures or self-managing their termination with non-recommended methods outside the formal healthcare system; this is particularly true in the context of legal restrictions. ${ }^{4}$ They present a measurement challenge, but more importantly, they 
put women at risk of abortion-related morbidity and mortality. In Nigeria, there are between 496 (95\% uncertainty interval (UI) 336 to 666) and 814 (95\% UI 596 to 1180) maternal deaths per 100000 live births, ${ }^{5-7}$ approximately $10 \%$ of which are due to unsafe abortion. ${ }^{8}$ Using the latest estimates of the general fertility rate, ${ }^{7}$ we estimate there are as many as 6000 abortion-related deaths annually, the majority of which are preventable. For each death, there are hundreds of women who experience severe and potentially life-threatening complications. ${ }^{9}$ Among gynaecological admissions at a Nigerian teaching hospital in recent years, $7.4 \%$ were related to treatment of unsafe abortion, $17 \%$ of which ultimately resulted in maternal death. ${ }^{9}$ Findings from gynaecological admissions at nine referral hospitals in Nigeria suggest that, although surgical abortion is still the primary method of abortion, the share of postabortion care (PAC) patients who report first using misoprostol is increasing. ${ }^{10}$ PAC patients who used misoprostol experienced fewer and less severe complications than PAC patients presenting after a clandestine surgical abortion. ${ }^{10}$ While these population level estimates and facility data are essential to track abortion trends and the public health implications of abortion, we know little about the specifics of its occurrence and characteristics of women who seek abortion, particularly those not seeking facility-based care.

Existing evidence indicate that the negative sequelae associated with unsafe abortion is experienced disproportionately by vulnerable women. ${ }^{411-13}$ A recent study in Nigeria found that young women aged 16-25 were the most likely to present for treatment of postabortion complications at a teaching hospital. ${ }^{9}$ Prior studies found that women experiencing abortion-related morbidities were younger, more likely to be unmarried and poorer than women experiencing maternal morbidities for other causes. ${ }^{11} 14$ However, these studies and much of the research in low-resource, legally restrictive settings have relied on facility-based, retrospective data, which are limited in the availability of information on women's characteristics and the generalisability of findings. In addition to the obvious burden of unsafe abortion morbidity and mortality on these women and their families, its treatment is associated with a significant cost to the public healthcare system. ${ }^{11} 14-16$

The first objective of this study is to estimate the 1-year incidence of induced abortion in Nigeria overall and by women's characteristics using direct report and the confidante methodology. ${ }^{17} 18$ The second objective is to determine the safety of reported abortions and its social determinants. The study complements facility-based studies of abortion in Nigeria, using a population-based approach to explore women's recent experiences of abortion within and outside of healthcare facilities.

\section{METHODS}

Sampling

Data for this study come from a population-based survey of reproductive age women (15-49) in Nigeria conducted by Performance Monitoring and Accountability 2020 (PMA2020) ${ }^{19}$ PMA2020 conducts frequent, low-cost and rapid turnaround national or regional surveys in several countries across Africa and Asia using smartphone technology ${ }^{19}{ }^{20}$ The Centre for Research, Evaluation Resources and Development is the implementing partner for PMA2020 in Nigeria while the Bill \& Melinda Gates Institute at the Johns Hopkins Bloomberg School of Public Health oversees the PMA2020 abortion measurement project and provides technical support.

PMA2020 surveys in Nigeria follow a three-stage cluster sampling design. First, seven states were selected using probability proportional to size (PPS) sampling: one state from five of the six geopolitical zones and two states from the North West zone, where $25 \%$ of Nigeria's total population resides. Within each state, geographic clusters defined as enumeration areas (EA) that contain approximately 200 households were selected using PPS sampling and subsequently 35-40 households were randomly selected per EA. Female resident interviewers invited all eligible female respondents ages 15 to 49 from the selected households to consent and participate in the face-to-face interview. This sampling strategy produced nationally representative samples of households and women of reproductive age in Nigeria. Data are also representative at the state level. For this study, we used data from PMA2020 Nigeria Round 5 collected between April and May 2018. The final sample included 11106 women. The Johns Hopkins Bloomberg School of Public Health and the National Health Research Ethics Committee of Nigeria provided ethical approval for this study. Women provided verbal consent prior to participation.

\section{Patient and public involvement}

No patients were involved in the development of the research questions and outcome measures.

\section{Measures}

The resident interviewers collected information about women's socioeconomic characteristics, their reproductive history, and their knowledge of and experience using contraception. In addition to these PMA2020 core questions, women also responded to an abortion module exploring the frequency, correlates and nature of abortion experiences in Nigeria.

The abortion module began with questions on the number of close female confidantes the respondent had, followed by questions on the age and highest level of education ever attended by the respondent's closest confidante. A confidant was defined as a woman aged 15-49, residing in Nigeria, and someone with whom the respondent reciprocally shares personal information. The interviewer then inquired about the closest confidante's experiences with abortion, specifically asking about pregnancy removal when pregnant or worried she was pregnant and separately about period regulation at a 
Table 1 Characteristics of Nigerian female respondents aged 15-49 and their closest female confidantes aged 15-49 who had a prior abortion*

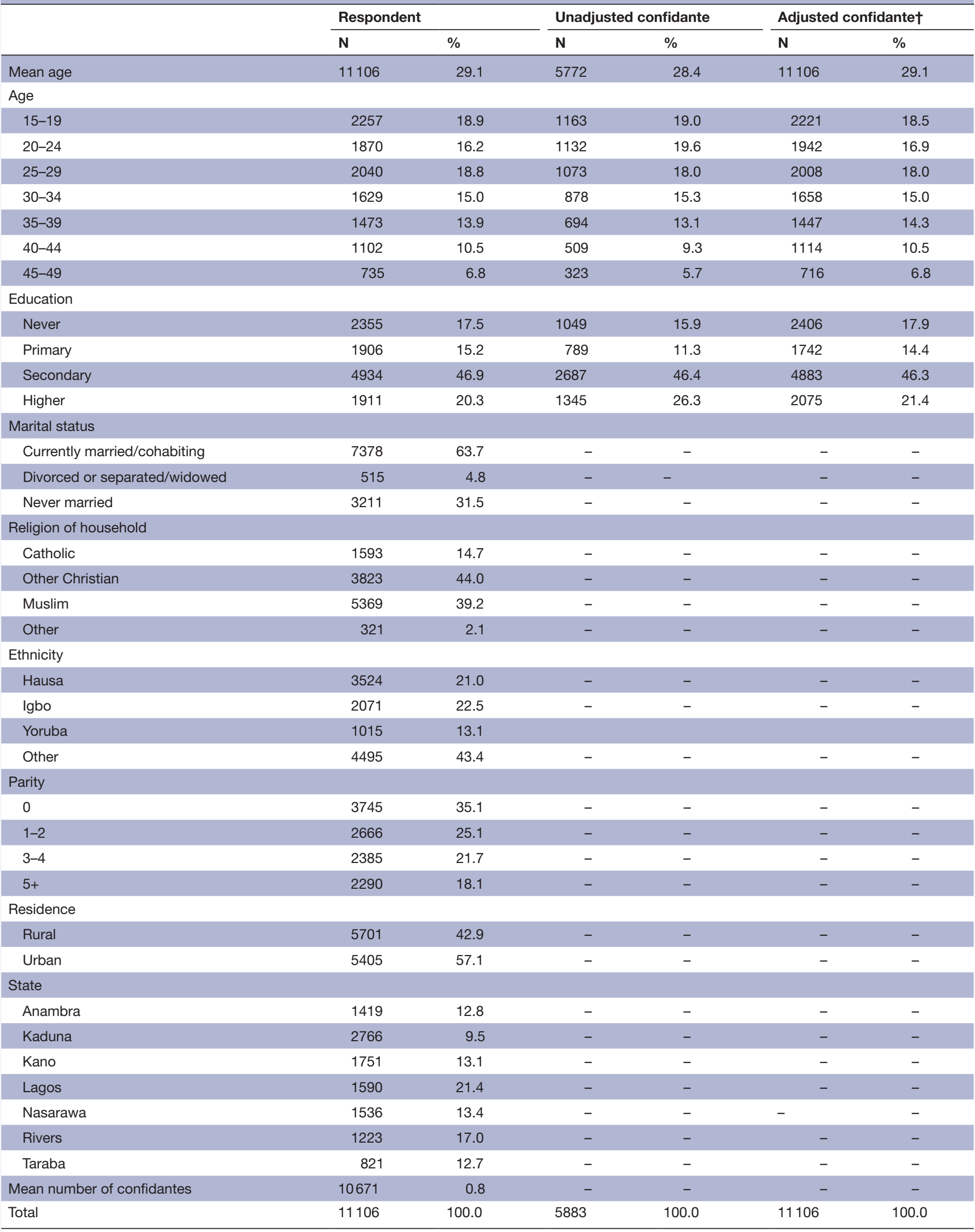

${ }^{*} \mathrm{Ns}$ across catgories of a characteristic that do not sum to total $\mathrm{N}$ is a result of missingness.

†Including respondent characteristics for confidantes and post-stratification weights. 
time when she was worried she was pregnant. This indirect approach, relying on respondent's reports of their closest friend's experience with abortion, builds off prior social network-based measurement of abortion. ${ }^{17}$ 21-24 Subsequent questions related to the respondent's own experiences with these phenomena. Other questions investigated pathways to confidantes' and respondents' abortions, including whether the women made multiple attempts to end the pregnancy or bring back a period, which method(s) she used, and the source(s) of these methods. If a woman reported doing multiple things to induce an abortion, subsequent questions asked about the first method and source followed by the last method and source. We categorised abortion methods intro surgery, medication abortion (MA) drugs, other pills or pills without sufficient information to categorise as MA, and traditional or other methods (like herbal drinks, injections, alcohol, or other traditional remedies). We categorised sources into public facility types, private facility types (including non-governmental organisations and private doctors), pharmacies or chemist shops and traditional or other non-medical sources (including shops, markets, friends or relatives or home).

Using these data, we operationalised abortion safety based on two dimensions: (1) whether the method(s) used included any non-recommended methods (ie, other than surgery or MA drugs) that put the woman at potentially high risk of abortion related morbidity or mortality and (2) whether the source(s) used were clinical (public or private facilities) or non-clinical (any other source). If a woman reported doing multiple things, we categorised her abortion as non-recommended if she used a method other than surgery or MA drugs at any point in the termination; we similarly categorised an abortion as non-clinical if at any point she used a source other than a public or private facility. We combined source and method information to categorise a woman's abortion into one of the following four safety categories: (1) recommended method(s) involving only clinical source(s); (2) recommended method involving nonclinical source(s); (3) non-recommended method(s) involving clinical source(s) and (4) non-recommended method(s) involving non-clinical source(s). Abortions in group four we deemed the most unsafe. We have described our safety measurement approach in more detail elsewhere. ${ }^{25}$

\section{Analyses}

For the analyses, we first examined the respondent characteristics and the limited demographic characteristics of the confidantes, which only included age and education. We calculated 1-year incidences of induced abortion by averaging the pregnancy removal incidence and the combined pregnancy removal/period regulation incidence separately for the respondent and confidante data. For both the respondents and confidantes, we took the average of the two point estimates as we believe the pregnancy removal data fails to capture some abortions while the period regulation data likely includes experiences that we would not consider to be abortions. In addition, we excluded any abortions where the woman used only emergency contraception and did not seek subsequent care assuming that the woman was not in fact pregnant (respondents $n=14$, confidantes $n=12$ ). For the confidante estimates, we included pregnancy removal and period regulations that the respondent reported with certainty ('Yes, I am certain') or with less certainty ('Yes, I think so') but for which she could provide details on the method(s) used. We made this adjustment to account for incomplete transmission of information on confidante abortions. ${ }^{18}$ For 'missing' confidantes (ie, those respondents who reported zero confidantes), we used a Poisson model to predict the likelihood of these 'missing' confidantes having had an abortion in the prior year. This involved regressing the respondent's socioeconomic characteristics on the available confidante abortion incidence data. This analytical approach adjusts for selection bias in the confidante sample. ${ }^{26}$ We then predicted the likelihood of the 'missing' confidantes having had a recent abortion using results of the Poisson regression. We combined the predicted likelihood for the 'missing' confidantes with the reported confidante incidence data to calculate the 1-year confidante abortion incidence estimates (separately for pregnancy removal and pregnancy removal/period regulation combined). To ensure these confidante data had characteristics that reflected the population of reproductive aged women in Nigeria, we constructed post-stratification weights using the weighted respondent data distributions as the reference. We used these adjusted data to calculate separate 1-year abortion incidences overall and by age, education, residence and state for respondents and by age and education for confidantes. We then conducted separate respondent and confidante bivariate and multivariable logistic regression analyses to determine which characteristics were independently associated with having reported a recent likely-abortion (pregnancy and period regulation combined) since we could not investigate these relationships for the average pregnancy removal and pregnancy removal/period regulation at the individual level. Additionally, the confidante data do not include the Poisson predicted estimates for the 'missing' confidantes since the outcome variable produced was no longer dichotomous, prohibiting logistic regression analyses that assume Bernoulli distribution.

With regard to safety, we first separately estimated the overall respondent and confidante distribution of abortion across the four safety categories among all reported likely-abortions (pregnancy removals and period regulations combined). We separately estimated the proportion of women who experienced the most unsafe abortions by background characteristics. Additionally, we conducted bivariate and multivariable logistic regressions to examine what characteristics were independently associated with 
increased odds of experiencing a most unsafe abortion. Unlike the confidante incidence estimate, we did not impute abortion safety for 'missing' confidantes in any of the associated analyses. Last, we calculated the 1-year incidence rate of most unsafe abortions and the corresponding annual number of most unsafe abortions in Nigeria.

We conducted all analyses in Stata V.15.1 $1^{27}$ and present results from weighted analyses that used the Taylor linearization approach to account for the complex sampling design and clustering.

\section{RESULTS}

\section{Sample characteristics}

A total of 11106 women of reproductive age completed the female survey (table 1). The 10671 respondents who answered the question on number of close female confidantes reported 0.8 confidantes on average and provided demographic and abortion experience details for their 5883 closest confidantes. Respondents were on average 29.1 years old, most had attended at least some secondary school $(46.9 \%)$ and the majority were currently married or cohabiting (63.7\%). Respondents primarily identified as Christian (not including Catholic) $(44.0 \%)$ or Muslim $(39.2 \%)$, and Igbo $(22.5 \%)$ or Hausa $(21.0 \%)$ ethnicity. Many respondents were nulliparous $(35.1 \%)$, yet nearly one in five had five or more children (18.1\%). Unadjusted confidante data indicate they were similar in age (average 28.4), but slightly more educated; $26.3 \%$ had higher education compared with $20.3 \%$ of respondents. Additionally, respondents in rural areas were more likely to report a confidante. After adjusting confidante data to account for respondents who did not report a confidante and applying the post-stratification weights, the distribution of confidante characteristics were no longer statistically significantly different from that of the respondents.

\section{Incidence estimates}

Overall, the 1-year induced likely-abortion incidence (pregnancy removal and period regulation combined) for respondents was 39.4 (SE 3.98) per 1000 women aged 15-49 while the adjusted confidante incidence was 56.5 (SE 2.91); removing the period regulations, the overall pregnancy removal incidence for respondents and confidantes were 18.7 (SE 1.92) and 35.1 (SE 2.04), respectively. To produce the final abortion incidence estimate, we averaged the pregnancy removal and combined pregnancy removal/period regulation rates separately for respondents and confidantes, which resulted in an estimated 29.0 (95\% CI 23.3 to 34.8) and 45.8 (95\% CI 41.0 to 50.6) abortions per 1000 women of reproductive age, respectively. All subsequent results are for the average pregnancy removal and period regulation incidences, which we refer to simply as abortion and present separately for respondents and confidantes.

The respondent and confidante abortion incidences revealed similar patterns by characteristics (figure 1).
Women aged 20-24 among respondents and confidantes had the highest 1-year abortion incidence at 47.9 and 77.6 abortions per 1000 women of reproductive age, respectively, followed by women aged 25-29 (40.8 and 58.0 ). Women in their $40 \mathrm{~s}$ had the lowest incidence for respondents and confidantes (13.5 and 25.5 among 40-44year olds and 8.9 and 23.0 among $45-49$ years olds, respectively). With respect to education, respondents and confidantes with secondary (34.8 and 57.1) or higher education (36.7 and 52.0) had the highest incidences of abortion and women who had never received formal education had the lowest (9.8 and 18.1). Respondents in rural areas reported significantly lower rates of abortion at 18.2 per 1000 women of reproductive age compared with 37.4 in urban areas. Women in Rivers state had the highest abortion rate among respondents (57.4) while women in Kano reported the lowest (5.4). The poorest respondents were least likely to have had a recent abortion (14.9). Examining the reporting ratio between the adjusted confidante data and the respondent data, we see that the youngest and oldest respondents were similarly more than two times as likely to underreport an abortion when asked directly about their own abortion experience, while respondents with no education were the most likely to underreport. Using the likely-abortion data, bivariate respondent results confirm patterns identified from examining incidences and were statistically significant (table 2); confidante bivariate results similarly indicated that being aged 20-24 and having secondary or higher education were significantly associated with increased odds of recent likely-abortion. Young age and urban residence remained significantly positively associated with abortion incidence in the multivariable respondent model while some states remained negatively associated. Confidante age and education both remained significant factors in the multivariable confidante model (table 2).

\section{Safety estimates}

A minority of respondents terminated their pregnancy using recommended methods $(34.5 \%)$ and only $31.2 \%$ received care from clinical sources; the corresponding figures based on confidante experiences were $29.5 \%$ and $23.9 \%$, respectively. Combining both criteria, most respondent (63.4\%) and confidante $(68.6 \%)$ likely-abortions fell into the most unsafe category, involving non-recommended methods dispensed by non-clinical or no providers (table 3). Few respondent and confidante likely-abortions were categorised as involving recommended method(s) dispensed in non-clinical settings $(5.4 \%$ vs $7.5 \%)$ or non-recommended method(s) dispensed by clinical source(s) $(2.1 \%$ vs $1.9 \%$ ), while only $29.1 \%$ and $22.0 \%$ of respondent and confidante likely-abortions were performed using recommended methods in a clinical setting. Compared with the overall safety distribution among all likely-abortions, those reported in the last 5 years were more likely to involve non-recommended methods from a non-clinical source for respondents $(73.6 \%)$ and confidantes $(72.2 \%)$ (estimates not shown). Using the national confidante abortion 


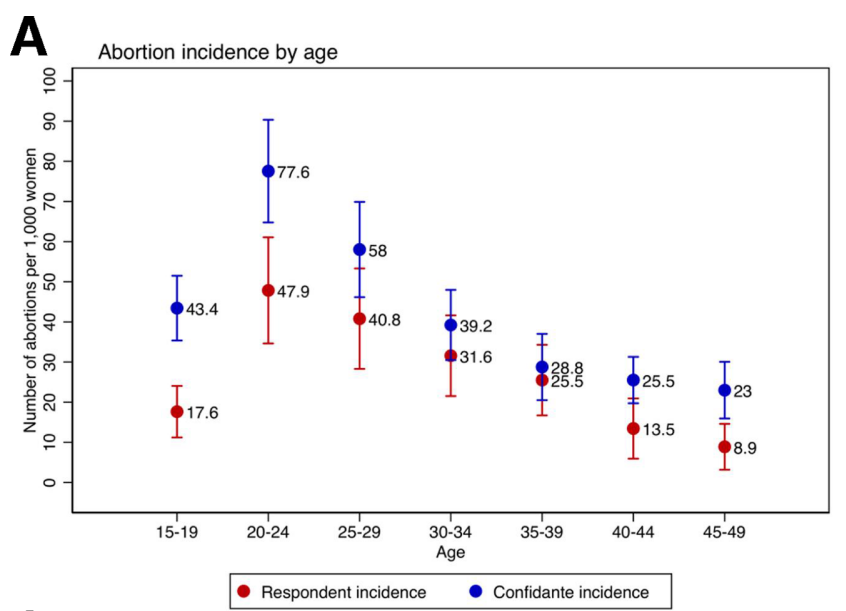

B
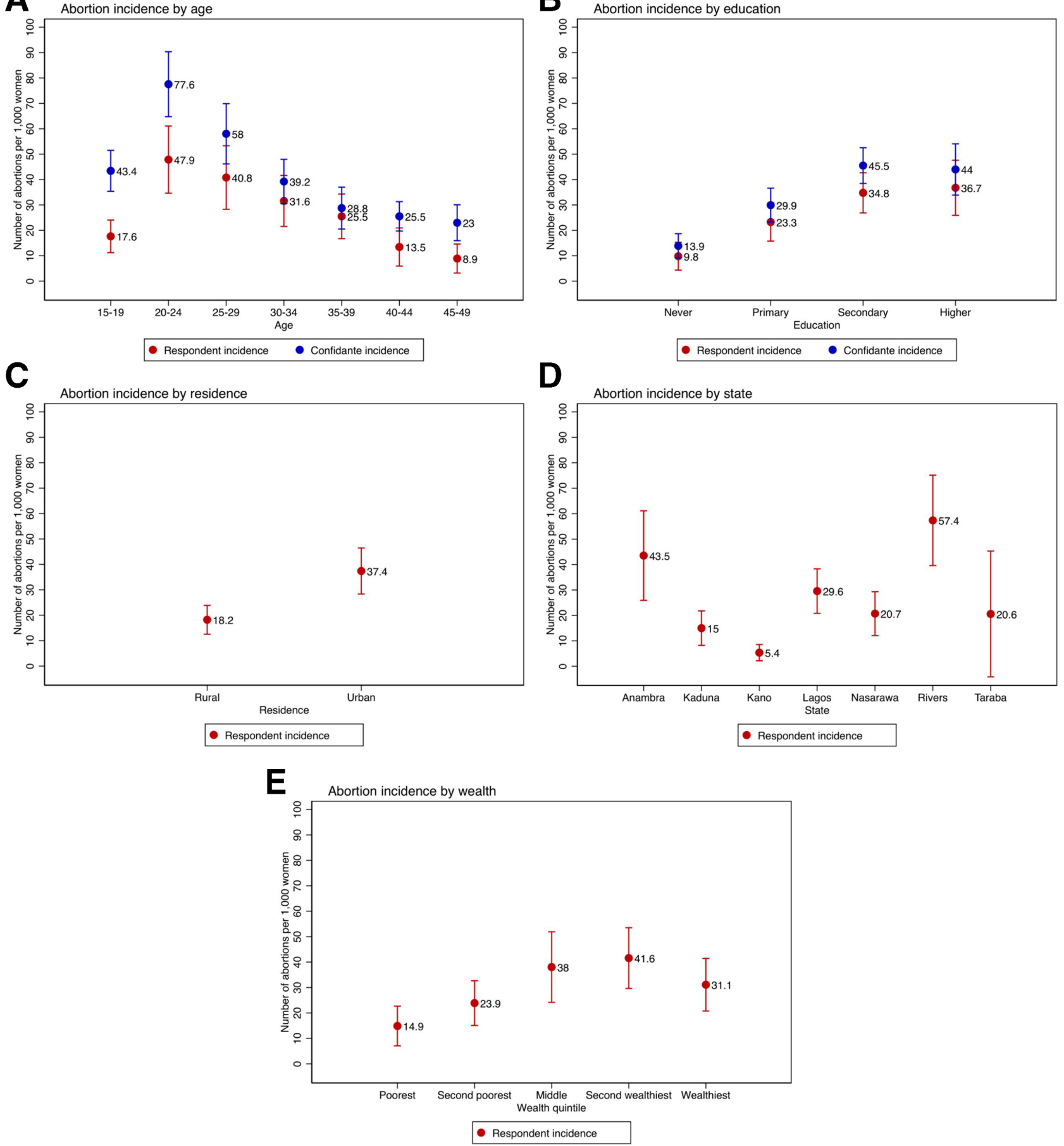

Figure 1 One-year incidence of abortion (average of pregnancy removal and combined pregnancy removal/period regulation rates) per 1,000 women of reproductive age among female respondents and their closest female confidantes in Nigeria by background characteristics.

incidence of 45.8, these safety estimates suggest an unsafe abortion rate of approximately 33 per 1000 women of reproductive age.

Examining likely-abortion safety by background characteristics, respondent and confidante results revealed similar patterns (figure 2). Women aged 15-19 were the most likely to have had the most unsafe abortions $(87.8 \%$ and $84.7 \%$ ), as were women who had never attended school $(79.1 \%$ and $86.4 \%)$. Respondents residing in rural areas were significantly more likely to have had the most unsafe likely-abortions $(70.8 \%)$. By state, respondents from Anambra, Kaduna, Nasarawa and Taraba had the highest levels of the most unsafe likely-abortions. Based on respondent data, the poorest women $(81.0 \%)$ were the most likely to have experienced the most unsafe likely-abortions. Results from a multivariable logistic regression indicated that age was independently associated with likely-abortion safety among respondents and 
Table 2 Bivariate and multivariate regressions of characteristics associated with experiencing an abortion in the year prior to the survey among Nigerian respondents and confidantes age 15-49*

\begin{tabular}{|c|c|c|c|c|c|c|c|c|c|c|c|c|}
\hline & \multicolumn{6}{|c|}{ Respondent $(n=11070)$} & \multicolumn{6}{|c|}{ Unadjusted confidante $(n=5883)$} \\
\hline & \multirow[t]{2}{*}{ OR } & \multicolumn{2}{|c|}{$95 \% \mathrm{Cl}$} & \multirow[t]{2}{*}{ aOR } & \multicolumn{2}{|c|}{$95 \% \mathrm{Cl}$} & \multirow[t]{2}{*}{ OR } & \multicolumn{2}{|c|}{$95 \% \mathrm{Cl}$} & \multirow[t]{2}{*}{ aOR } & \multicolumn{2}{|c|}{$95 \% \mathrm{Cl}$} \\
\hline Age & & & & & & & & & & & & \\
\hline $15-19$ & 1.00 & - & - & 1.00 & - & - & 1.00 & - & - & 1.00 & - & - \\
\hline $20-24$ & 2.93 & 2.08 & 4.13 & 2.89 & 2.07 & 4.05 & 1.93 & 1.17 & 3.19 & 2.18 & 1.30 & 3.65 \\
\hline $25-29$ & 2.38 & 1.62 & 3.49 & 2.15 & 1.43 & 3.22 & 1.43 & 0.81 & 2.52 & 1.63 & 0.94 & 2.83 \\
\hline $30-34$ & 1.66 & 1.11 & 2.49 & 1.47 & 0.99 & 2.19 & 0.84 & 0.44 & 1.57 & 0.99 & 0.53 & 1.85 \\
\hline 35-39 & 1.38 & 0.90 & 2.12 & 1.24 & 0.80 & 1.93 & 0.94 & 0.52 & 1.72 & 1.14 & 0.61 & 2.12 \\
\hline $40-44$ & 0.65 & 0.35 & 1.23 & 0.66 & 0.35 & 1.23 & 0.44 & 0.18 & 1.06 & 0.54 & 0.22 & 1.33 \\
\hline $45-49$ & 0.62 & 0.32 & 1.17 & 0.58 & 0.30 & 1.10 & 0.43 & 0.17 & 1.12 & 0.54 & 0.21 & 1.39 \\
\hline \multicolumn{13}{|l|}{ Education } \\
\hline Never & 1.00 & - & - & 1.00 & - & - & 1.00 & - & - & 1.00 & - & - \\
\hline Primary & 2.47 & 1.44 & 4.26 & 1.57 & 0.84 & 2.92 & 1.76 & 0.79 & 3.93 & 1.80 & 0.80 & 4.06 \\
\hline Secondary & 3.60 & 2.08 & 6.24 & 1.51 & 0.76 & 3.00 & 3.51 & 1.83 & 6.70 & 3.40 & 1.73 & 6.70 \\
\hline Higher & 4.02 & 2.28 & 7.08 & 1.62 & 0.81 & 3.25 & 2.66 & 1.29 & 5.45 & 2.52 & 1.25 & 5.06 \\
\hline \multicolumn{13}{|l|}{ Residence } \\
\hline Rural & 1.00 & - & - & 1.00 & - & - & - & - & - & - & - & - \\
\hline Urban & 2.23 & 1.47 & 3.40 & 2.05 & 1.18 & 3.55 & - & - & - & - & - & - \\
\hline \multicolumn{13}{|l|}{ State } \\
\hline Anambra & 1.00 & - & - & 1.00 & - & - & - & - & - & - & - & - \\
\hline Kaduna & 0.37 & 0.21 & 0.64 & 0.39 & 0.19 & 0.80 & - & - & - & - & - & - \\
\hline Kano & 0.10 & 0.05 & 0.20 & 0.12 & 0.05 & 0.27 & - & - & - & - & - & - \\
\hline Lagos & 0.75 & 0.48 & 1.19 & 0.68 & 0.42 & 1.10 & - & - & - & - & - & - \\
\hline Nasarawa & 0.46 & 0.27 & 0.80 & 0.58 & 0.26 & 1.29 & - & - & - & - & - & - \\
\hline Rivers & 1.34 & 0.81 & 2.22 & 1.36 & 0.84 & 2.20 & - & - & - & - & - & - \\
\hline Taraba & 0.60 & 0.15 & 2.43 & 0.79 & 0.11 & 5.43 & - & - & - & - & - & - \\
\hline \multicolumn{13}{|l|}{ Wealth quintile } \\
\hline Poorest & 1.00 & - & - & 1.00 & - & - & - & - & - & - & - & - \\
\hline Second poorest & 1.74 & 0.88 & 3.45 & 0.98 & 0.43 & 2.24 & - & - & - & - & - & - \\
\hline Middle & 2.73 & 1.38 & 5.39 & 1.02 & 0.37 & 2.83 & - & - & - & - & - & - \\
\hline Second wealthiest & 2.70 & 1.43 & 5.12 & 0.89 & 0.32 & 2.47 & - & - & - & - & - & - \\
\hline Wealthiest & 2.11 & 1.08 & 4.13 & 0.64 & 0.22 & 1.83 & - & - & - & - & - & - \\
\hline
\end{tabular}

${ }^{*}$ Bold values indicate statistical significance at the $p<0.05$ level.

aOR, Adjusted odds ratio; OR, Odds ratio.

confidantes, with older age associated with lower likelihood of most unsafe likely-abortion (table 4). Greater confidante education remained significantly associated with lower odds of a most unsafe likely-abortion while it was no longer significant among respondents, for whom we were able to adjust for wealth. For respondents, some states and wealth quintiles significantly associated with lower odds of having experienced a most unsafe likelyabortion in the multivariable model, with increasing wealth associated with decreasing odds (table 4).

\section{DISCUSSION}

Results from this study provide new insights regarding the frequency, correlates and conditions under which women have abortions in Nigeria. Respondent and confidante incidences both indicate that abortion in this setting is common and more likely to be reported among young, educated women while respondent results also suggest greater incidence among urban women. Given the concerns with underreporting of self-reported abortion, ${ }^{28-30}$ the respondent 1-year incidence of pregnancy removal (18.7) is likely an underestimate, while the corresponding confidante estimate of 35.2 may also be an underestimate if it fails to capture experiences that women do not consider to be abortions and instead report as period regulations. Compared with the Bankole et als Abortion Incidence Complications Methodology (AICM) study estimate of 33 abortions per 1000 women aged 15-49 in 2012, 
Table 3 Safety of most recent reported likely-abortion among female respondents aged 15-49 and their closest female confidantes aged 15-49 in Nigeria

\begin{tabular}{|c|c|c|c|c|}
\hline & \multicolumn{2}{|c|}{ Respondent } & \multicolumn{2}{|c|}{ Confidante* } \\
\hline & Estimate & $\mathbf{N}$ & Estimate & $\mathbf{N}$ \\
\hline $\begin{array}{l}\text { Recommended method, } \\
\text { clinical provider }\end{array}$ & 29.1 & 471 & 22.0 & 266 \\
\hline $\begin{array}{l}\text { Recommended method, } \\
\text { non-clinical provider }\end{array}$ & 5.4 & 97 & 7.5 & 101 \\
\hline $\begin{array}{l}\text { Non-recommended } \\
\text { method, clinical provider }\end{array}$ & 2.1 & 37 & 1.9 & 29 \\
\hline $\begin{array}{l}\text { Non-recommended } \\
\text { method, non-clinical } \\
\text { provider }\end{array}$ & 63.4 & 1196 & 68.6 & 969 \\
\hline Total & 100.0 & 1810 & 100.0 & 1370 \\
\hline
\end{tabular}

*Bold values indicate statistically significantly different at the $\mathrm{p}<0.05$ level (reference respondent).

our self-reported pregnancy removal estimate is lower while our confidante estimate is similar. ${ }^{1}$ Experiences captured via the pregnancy removal questions may be most analogous to the abortion experiences captured in an AICM study while reported period regulations may include some non-abortions. However, we believe the true 1-year abortion incidence in Nigeria is between the confidante pregnancy removal rate and the confidante combined pregnancy removal/period regulation rate. Our final point estimate averages the high and low values of the confidante range, resulting in a national 1-year incidence of 45.8 abortions per 1000 women of reproductive age.

Even if some of the period regulations captured in our data were not abortions, to the extent that they identify women taking potentially harmful actions post-coitally to bring back their menses at a time when they were worried they were pregnant, we are concerned about them from a public health perspective. Given the greater visibility of unsafe abortions that result in complications, we believe the confidante safety estimates are biased upward. As such, we think the respondent safety estimates are closer to the truth. Respondent findings indicate that $63.4 \%$ of likely-abortions in Nigeria are most unsafe while $73.7 \%$ of those that occurred in the prior 5 years were most unsafe; this is lower than the recent unsafe abortion estimates for Western Africa in 2010-2014 (84.7\%). ${ }^{3}$ This may be because we exclude all clinical sources from the most unsafe category; however, we know many abortions in facilities are performed under unsafe conditions.

Our safety-related findings indicate that the majority of likely-abortions were the most unsafe, with younger, poorer and less educated women at greatest risk of having unsafe abortions. Multivariable results suggest that wealth may be the determining factor in whether a woman undergoes a most unsafe abortion. These results are consistent with previous literature suggesting that the most disadvantaged women are those most likely to resort to unsafe means of termination. ${ }^{411}{ }^{14}$ Evidence from this study confirms that abortion in Nigeria is a public health concern and an issue of social inequity.

This study has limitations. Most importantly, we were unable to validate the abortion estimates against an external, objective measure. Thus, while we view the confidante estimates as more accurate than the respondents', we do not know by how much. Stigma and legal restrictions surrounding abortion in Nigeria (and evidence of incomplete transmission of respondent abortions to their confidantes, which we present elsewhere ${ }^{18}$ ) suggest that all confidantes who had an abortion had not told their corresponding respondents. However, our inclusion of the confidante abortions that respondents reported with less certainty acts to counteract this possibility. Had we excluded all less certain respondent reported confidante abortions, the rate would be similar to the respondent rate. Beyond the issue of transmission, the surrogate sample of confidantes may bias estimates of abortion if their sociodemographic characteristics are different from the index population and such differences are correlated with the likelihood of abortion. ${ }^{26}$ In our study, $47.0 \%$ of women reported no female confidante. We adjusted for this potential bias by using the Poisson prediction approach, which decreased the confidante incidence estimate by $6.1 \%$, but biases may remain. Further work is needed to determine the optimal friend definition that minimises confidante sample selection bias while maximising confidante abortion experience transmission to respondents. Additionally, differential under-reporting could bias our bivariate and multivariate findings. Nonetheless, the patterns of abortion incidence and safety were similar for respondents and confidantes, which lends credibility to the conclusions regarding which groups of women are most likely to have an abortion or most unsafe abortion.

With regard to abortion safety measurement, the potential for differential underreporting by method and source is the primary limitation of the data that would lead to bias. Since the overall distribution and sociodemographic correlates of the most unsafe likely-abortions were similar among respondents and confidantes, we believe this potential bias is unlikely to be significant or to qualitatively affect our conclusions. Another limitation is the potential for misclassification. Women sometimes could not provide sufficient information for interviewers to classify the specific pill used, nor could they distinguish the specific conditions under which the surgery was performed. However, this limitation would have led to misclassifications in both directions (eg, pills recorded as 'other pills' that were actually MA drugs, or surgery in a facility that was actually performed by an untrained provider), limiting the extent of systematic error in the overall estimates.

The study has a number of strengths. The data are from a large, nationally representative survey. Investigators took extensive efforts during the questionnaire development and pilot testing to appropriately capture the nuance in how women discuss and refer to abortion experiences locally; this led to the different sets of questions about 

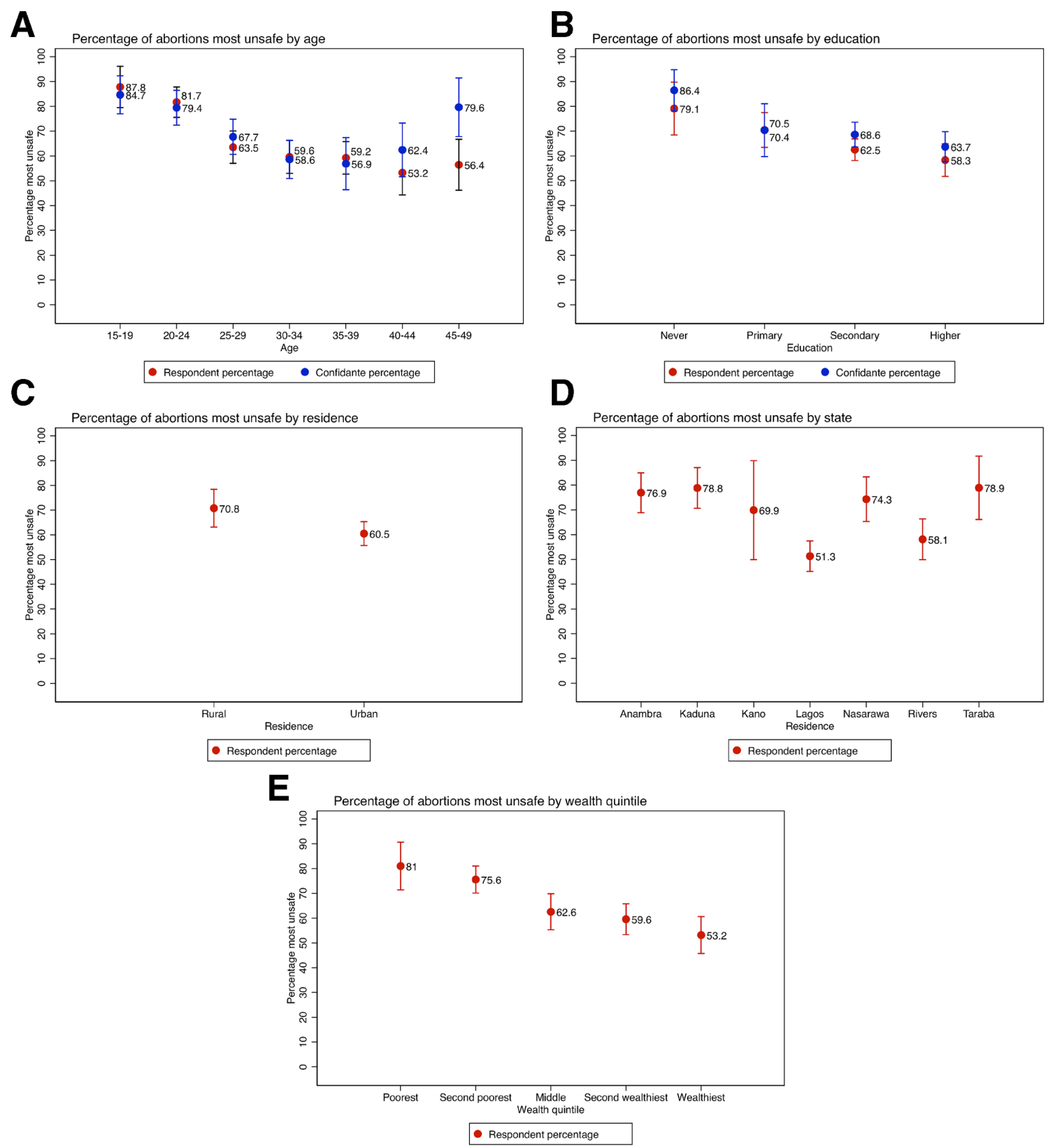

Figure 2 Percentage of most recent abortions among female respondents and their closest female confidantes in Nigeria that were the most unsafe by background characteristics.

pregnancy removal and period regulation. The methodological approach enabled estimation of abortion incidence and safety overall and by women's background characteristics, providing details on the characteristics of women most likely to have had an abortion or an unsafe abortion. Additionally, the use of both respondent and confidante data provided contemporaneous estimates of these abortion-related measures. Having data on both populations confirmed the usefulness of this social-network based indirect methodology in reducing the social desirability pressure and producing more accurate estimates while providing two sources of support regarding the patterns of abortion incidence and safety by women's characteristics.

\section{CONCLUSION}

The confidante data from this study provide a national 1-year induced abortion incidence of 45.8 per 1000 women of reproductive age, suggesting that abortion is significantly more common than previously estimated. Findings indicate that vulnerable, economically disadvantaged women with limited ability to navigate and access safe abortion in this legally restrictive setting are most at risk of having an unsafe abortion. Efforts to expand the legal conditions for abortion in Nigeria are critical as restrictive abortion laws negatively impact abortion safety without reducing overall abortion incidence rates. ${ }^{31}$ In the absence of legal expansion, women will continue to seek services from providers who are not regulated and may not have appropriate training but are motivated to 
Table 4 Multivariate regression of characteristics associated with experiencing a most unsafe abortion among Nigerian respondents and confidantes aged $15-49^{*}$

\begin{tabular}{|c|c|c|c|c|c|c|c|c|c|c|c|c|}
\hline \multirow{3}{*}{ Age } & \multicolumn{6}{|c|}{ Respondent $(n=1801)$} & \multicolumn{6}{|c|}{ Confidante $(n=1365)$} \\
\hline & \multirow[t]{2}{*}{ OR } & \multicolumn{2}{|c|}{$95 \% \mathrm{Cl}$} & \multirow[t]{2}{*}{ aOR } & \multicolumn{2}{|l|}{$95 \% \mathrm{Cl}$} & \multirow[t]{2}{*}{ OR } & \multicolumn{2}{|c|}{$95 \% \mathrm{Cl}$} & \multirow[t]{2}{*}{ aOR } & \multicolumn{2}{|c|}{$95 \% \mathrm{Cl}$} \\
\hline & & & & & & & & & & & & \\
\hline $15-19$ & 1.00 & - & - & 1.00 & - & - & 1.00 & - & - & 1.00 & - & - \\
\hline $20-24$ & 0.62 & 0.26 & 1.49 & 0.62 & 0.26 & 1.51 & 0.70 & 0.38 & 1.38 & 0.73 & 0.36 & 1.45 \\
\hline $25-29$ & 0.24 & 0.11 & 0.53 & 0.28 & 0.12 & 0.62 & 0.37 & 0.19 & 0.73 & 0.41 & 0.21 & 0.80 \\
\hline $30-34$ & 0.21 & 0.09 & 0.49 & 0.25 & 0.10 & 0.60 & 0.25 & 0.12 & 0.47 & 0.26 & 0.13 & 0.51 \\
\hline $35-39$ & 0.20 & 0.09 & 0.47 & 0.21 & 0.09 & 0.50 & 0.23 & 0.12 & 0.47 & 0.23 & 0.11 & 0.47 \\
\hline $40-44$ & 0.16 & 0.07 & 0.36 & 0.17 & 0.07 & 0.38 & 0.31 & 0.15 & 0.63 & 0.31 & 0.15 & 0.64 \\
\hline $45-49$ & 0.18 & 0.08 & 0.43 & 0.19 & 0.08 & 0.44 & 0.69 & 0.26 & 1.86 & 0.72 & 0.27 & 1.91 \\
\hline \multicolumn{13}{|l|}{ Education } \\
\hline Never & 1.00 & - & - & 1.00 & - & - & 1.00 & - & - & 1.00 & - & - \\
\hline Primary & 0.63 & 0.33 & 1.21 & 0.77 & 0.41 & 1.47 & 0.37 & 0.17 & 0.81 & 0.36 & 0.16 & 0.80 \\
\hline Secondary & 0.44 & 0.22 & 0.87 & 0.56 & 0.28 & 1.12 & 0.34 & 0.16 & 0.71 & 0.30 & 0.15 & 0.63 \\
\hline Higher & 0.37 & 0.19 & 0.74 & 0.64 & 0.31 & 1.33 & 0.28 & 0.13 & 0.58 & 0.29 & 0.14 & 0.61 \\
\hline \multicolumn{13}{|l|}{ Residence } \\
\hline Rural & 1.00 & - & - & 1.00 & - & - & - & - & - & - & - & - \\
\hline Urban & 0.63 & 0.41 & 0.97 & 1.23 & 0.80 & 1.89 & - & - & - & - & - & - \\
\hline \multicolumn{13}{|l|}{ State } \\
\hline Anambra & 1.00 & - & - & 1.00 & - & - & - & - & - & - & - & - \\
\hline Kaduna & 1.12 & 0.57 & 2.19 & 0.61 & 0.31 & 1.22 & - & - & - & - & - & - \\
\hline Kano & 0.70 & 0.24 & 2.00 & 0.37 & 0.12 & 1.12 & - & - & - & - & - & - \\
\hline Lagos & 0.32 & 0.19 & 0.53 & 0.31 & 0.18 & 0.54 & - & - & - & - & - & - \\
\hline Nasarawa & 0.87 & 0.45 & 1.67 & 0.41 & 0.19 & 0.87 & - & - & - & - & - & - \\
\hline Rivers & 0.42 & 0.24 & 0.73 & 0.35 & 0.20 & 0.61 & - & - & - & - & - & - \\
\hline Taraba & 1.12 & 0.46 & 2.75 & 0.54 & 0.21 & 1.43 & - & - & - & - & - & - \\
\hline \multicolumn{13}{|l|}{ Wealth quintile } \\
\hline Poorest & 1.00 & - & - & 1.00 & - & - & - & - & - & - & - & - \\
\hline Second poorest & 0.73 & 0.37 & 1.41 & 0.82 & 0.42 & 1.59 & - & - & - & - & - & - \\
\hline Middle & 0.39 & 0.19 & 0.79 & 0.46 & 0.22 & 0.98 & - & - & - & - & - & - \\
\hline Second wealthiest & 0.35 & 0.18 & 0.68 & 0.43 & 0.21 & 0.91 & - & - & - & - & - & - \\
\hline Wealthiest & 0.27 & 0.13 & 0.53 & 0.35 & 0.16 & 0.77 & - & - & - & - & - & - \\
\hline
\end{tabular}

${ }^{*}$ Bold values indicate statistical significance at the $p<0.05$ level.

provide abortion for financial gain ${ }^{32}$ and/or to alleviate suffering associated with unwanted pregnancies. In the meantime, some local organisations are currently implementing harm reduction efforts to increase awareness of MA drugs to more safely self-induce, which can help mitigate the toll of abortion-related morbidity and mortality. Additionally, improved availability of contraceptive services, including counselling to counteract fears of contraceptive-related infertility, ${ }^{33}$ is needed to reduce women's reliance on unsafe abortion alone to control their fertility. More broadly, reproductive health policies and programmes must work to ensure equitable access to contraceptive and safe abortion services for legal indications. Furthermore, availability of quality PAC is critically needed to reduce abortion-related morbidity and mortality given the frequent recourse to unsafe abortion.
Inadequate action on any of these fronts will result in continued preventable deaths from unsafe abortion.

Correction notice This article has been corrected since it was published. The article type has been updated.

Contributors SB, CM and MS conceived the study and led development of the study instrument. EO and FO led in-country data collection and provided input on the study instrument. SB and MS led the data analysis with input from FO, EO and $\mathrm{CM}$. All authors were involved in the manuscript writing and provided final approval of the manuscript.

Funding This study was funded by an Anonymous Donor (Grant number: 127941). Competing interests None declared.

Patient consent for publication Not required.

Provenance and peer review Not commissioned; externally peer reviewed.

Data availability statement Data are available in a public, open access repository. 
Open access This is an open access article distributed in accordance with the Creative Commons Attribution Non Commercial (CC BY-NC 4.0) license, which permits others to distribute, remix, adapt, build upon this work non-commercially, and license their derivative works on different terms, provided the original work is properly cited, appropriate credit is given, any changes made indicated, and the use is non-commercial. See: http://creativecommons.org/licenses/by-nc/4.0/.

\section{ORCID iD}

Caroline Moreau http://orcid.org/0000-0002-8637-6249

\section{REFERENCES}

1 Bankole A, Adewole I, Hussain R, et al. The incidence of abortion in Nigeria. Int Perspect Sex Reprod Health 2015;41:170-81.

2 Henshaw SK, Singh S, Oye-Adeniran BA, et al. The incidence of induced abortion in Nigeria. Int Fam Plan Perspect 1998;24:156-64.

3 Ganatra B, Gerdts C, Rossier C, et al. Global, regional, and subregional classification of abortions by safety, 2010-14: estimates from a Bayesian hierarchical model. The Lancet 2017;390:2372-81.

4 Singh S, Remez L, Sedgh G, et al. Abortion worldwide 2017: uneven progress and unequal access abortion worldwide 2017: uneven progress and unequal access. 2018.

5 Kassebaum NJ, Bertozzi-Villa A, Coggeshall MS, et al. Global, regional, and national levels and causes of maternal mortality during 1990-2013: a systematic analysis for the global burden of disease study 2013. The Lancet 2014;384:980-1004.

6 World Health Organization. Trends in maternal mortality: 1990-2015: estimates from who, UNICEF, UNFPA, world bank group and the United nations population division: Executive summary. World Health Organization, 2015.

7 National Population Commission (NPC) [Nigeria], ICF International Nigeria demographic and health survey 2013. Abuja, Nigeria and Rockville, Maryland, USA: NPC and ICF International, 2014.

8 Say L, Chou D, Gemmill A, et al. Global causes of maternal death: a who systematic analysis. Lancet Glob Health 2014;2:e323-33.

9 Akinlusi FM, Rabiu KA, Adewunmi AA, et al. Complicated unsafe abortion in a Nigerian teaching hospital: pattern of morbidity and mortality. J Obstet Gynaecol 2018;38:961-6.

10 Bello FA, Fawole B, Oluborode B, et al. Trends in misoprostol use and abortion complications: a cross-sectional study from nine referral hospitals in Nigeria. PLoS One 2018;13:e0209415.

11 Prada E, Bankole A, Oladapo OT, et al. Maternal near-miss due to unsafe abortion and associated short-term health and socio-economic consequences in Nigeria. Afr J Reprod Health 2015;19:52-62.

12 Sundaram A, Vlassoff M, Mugisha F, et al. Documenting the individual- and household-level cost of unsafe abortion in Uganda. Int Perspect Sex Reprod Health 2013;39:174-84.

13 Sundaram A, Juarez F, Bankole A, et al. Factors associated with Abortion-Seeking and obtaining a safe abortion in Ghana. Stud Fam Plann 2012;43:273-86.

14 Henshaw SK, Adewole I, Singh S, et al. Severity and cost of unsafe abortion complications treated in Nigerian hospitals. Int Fam Plan Perspect 2008;34:040-51.

15 Vlassoff M, Walker D, Shearer J, et al. Estimates of health care system costs of unsafe abortion in Africa and Latin America. Int Perspect Sex Reprod Health 2009;35:114-21.
16 Bankole A, Singh S, Vlassoff M, et al. Chapter 4: Estimating the Cost of Post-Abortion Care in Nigeria: A Case Study. In: Lule E, Singh S, Chowdhury SA, et al, eds. Fertility regulation behaviors and their costs: contraception and unintended pregnancies in Agrica and eastern Europe and central Asia. Washington, DC: World Bank, 2007.

17 Sedgh G, Keogh SC. Novel approaches to estimating abortion incidence. Reprod Health 2019;16:44.

18 Bell SO, Shankar M, Omoluabi E, et al. Methodological Advances in Survey-Based Abortion Estimation: Promising Findings from Nigeria, India, and Cote d'Ivoire. Austin, Texas: Population Association of America Annual Meeting, 2019.

19 Performance Monitoring and Accountability 2020 (PMA2020). Performance monitoring and accountability 2020 (PMA2020) website, 2019. Available: https://www.pma2020.org/surveymethodology

20 Zimmerman L, Olson H, Tsui A, et al. PMA2020: rapid Turn-Around survey data to monitor family planning service and practice in ten countries. Stud Fam Plann 2017;48:293-303.

21 Grossman D, Hendrick E, Fuentes L, et al. Knowledge, opinion and experience related to abortion self-induction in Texas. Contraception 2015;92:360-1

22 Rossier C, Guiella G, Ouédraogo A, et al. Estimating clandestine abortion with the confidants method-results from Ouagadougou, Burkina Faso. Soc Sci Med 2006;62:254-66.

23 Sedgh G, Rossier C, Kaboré I, et al. Estimating abortion incidence in Burkina Faso using two methodologies. Stud Fam Plann 2011;42:147-54.

24 Yeatman S, Trinitapoli J. Best-friend reports: a tool for measuring the prevalence of sensitive behaviors. Am J Public Health 2011;101:1666-7.

25 Bell SO, OlaOlorun F, Shankar M, et al. Measurement of abortion safety using community-based surveys: findings from three countries. PLoS One 2019;14:e0223146-14.

26 Helleringer S, Adams Jimi, Yeatman S, et al. Evaluating sampling biases from third-party reporting as a method for improving survey measures of sensitive behaviors. Soc Networks 2019;59:134-40.

27 StataCorp. Stata 15 base reference manual. College Station, TX: Stata Press, 2017.

28 Jones RK, Kost K. Underreporting of induced and spontaneous abortion in the United States: an analysis of the 2002 national survey of family growth. Stud Fam Plann 2007;38:187-97.

29 Anderson BA, Katus K, Puur A, et al. The validity of survey responses on abortion: evidence from Estonia. Demography 1994;31:115-32.

30 Udry JR, Gaughan M, Schwingl PJ, et al. A medical record linkage analysis of abortion underreporting. Fam Plann Perspect 1996;28:228-31.

31 Sedgh G, Bearak J, Singh S, et al. Abortion incidence between 1990 and 2014: global, regional, and subregional levels and trends. The Lancet 2016;388:258-67.10.1016/S0140-6736(16)30380-4

32 Ordinioha B, Brisibe S. Clandestine abortion in Port Harcourt: providers' motivations and experiences. Niger J Med 2008;17:291-5.

33 Otoide VO, Oronsaye F, Okonofua FE. Why Nigerian adolescents seek abortion rather than contraception: evidence from focus-group discussions. Int Fam Plan Perspect 2001;27:77-81. 